\title{
Non-separating Planar Graphs
}

\author{
Hooman R. Dehkordi Graham Farr \\ Faculty of Information Technology \\ Monash University \\ Clayton, Victoria 3800, Australia \\ \{hooman.dehkordi, graham.farr\}@monash.edu
}

Submitted: Jun 23, 2019; Accepted: Dec 7, 2020; Published: Jan 15, 2021

(C) The authors. Released under the CC BY license (International 4.0).

\begin{abstract}
A graph $G$ is a non-separating planar graph if there is a drawing $D$ of $G$ on the plane such that (1) no two edges cross each other in $D$ and (2) for any cycle $C$ in $D$, any two vertices not in $C$ are on the same side of $C$ in $D$.

Non-separating planar graphs are closed under taking minors and are a subclass of planar graphs and a superclass of outerplanar graphs.

In this paper, we show that a graph is a non-separating planar graph if and only if it does not contain $K_{1} \cup K_{4}$ or $K_{1} \cup K_{2,3}$ or $K_{1,1,3}$ as a minor.

Furthermore, we provide a structural characterisation of this class of graphs. More specifically, we show that any maximal non-separating planar graph is either an outerplanar graph or a wheel or it is a graph obtained from the disjoint union of two triangles by adding three vertex-disjoint paths between the two triangles.

Lastly, to demonstrate an application of non-separating planar graphs, we use the characterisation of non-separating planar graphs to prove that there are maximal linkless graphs with $3 n-3$ edges. Thus, maximal linkless graphs can have significantly fewer edges than maximum linkless graphs; Sachs exhibited linkless graphs with $n$ vertices and $4 n-10$ edges (the maximum possible) in 1983 .
\end{abstract}

Mathematics Subject Classifications: 05C10, 05C83

\section{Introduction}

A drawing of a graph in the plane consists of a set of points representing (under a bijection) the vertices of the graph and a set of curves between certain pairs of points representing edges between corresponding vertex pairs of the graph where the curves do not pass through the points that represent vertices. A planar drawing is a drawing in which edges do not intersect.

Let $C$ be a cycle in a planar drawing $D$ of a graph $G$, then $C$ is a separating cycle if there is at least one vertex in the interior of $C$ and one vertex in the exterior of $C$. 

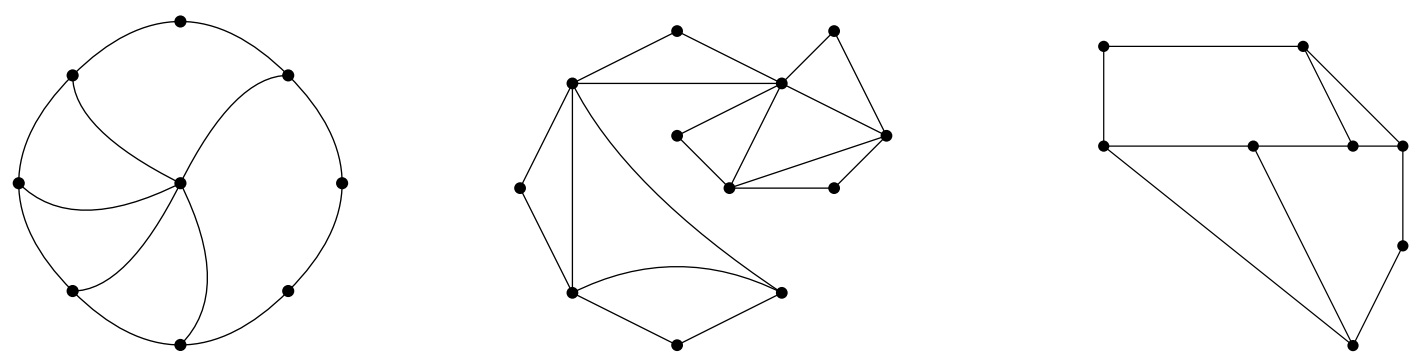

Figure 1: Three examples of non-separating planar graphs

A non-separating planar drawing of a graph is a planar drawing of the graph that does not contain any separating cycles. A non-separating planar graph is a graph that has a non-separating planar drawing (see for example Figure 1).

Any graph $G^{\prime}$ that can be obtained from a graph $G$ by a series of edge deletions, vertex deletions and edge contractions is called a minor of $G$.

A set $S$ of graphs is a minor-closed set or minor-closed family of graphs if any minor of a graph $G \in S$ is also a member of $S$.

In this paper we characterise non-separating planar graphs. This class is a subclass of planar graphs and a superclass of outerplanar graphs and is closed under minors. To characterise non-separating planar graphs we prove Theorems 1 and 2 .

Theorem 1. For any graph $G$, the following are equalivalent:

- $G$ is non-separating planar graphs,

- $G$ does not contain any of $K_{1} \cup K_{4}$ or $K_{1} \cup K_{2,3}$ or $K_{1,1,3}$ as a minor,

- $G$ does not contain any of $K_{1} \cup K_{4}$ or $K_{1} \cup K_{2,3}$ or $K_{1,1,3}$ as a subdivision ${ }^{1}$ (see Figure 2).

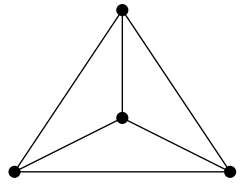

(a) $K_{1} \cup K_{4}$

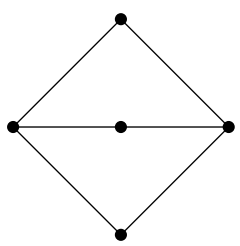

(b) $K_{1} \cup K_{2,3}$

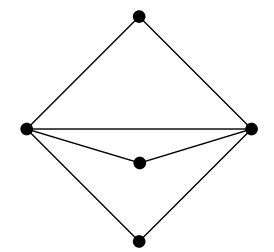

(c) $K_{1,1,3}$

Figure 2: Excluded minors for non-separating planar graphs

An edge $e=(u, v)$ in a graph $G$ is subdivided by replacing it with two edges $(u, w),(w, v)$ where $w$ is not a vertex of $G$. A subdivision of a graph $G$ is a graph that can be obtained by some sequence of subdivisions, starting with $G$. A graph is a triangular prism if it is isomorphic to the graph that is depicted in Figure 3a. A graph is an elongated triangular

\footnotetext{
${ }^{1}$ where $\cup$ denotes the disjoint union
} 


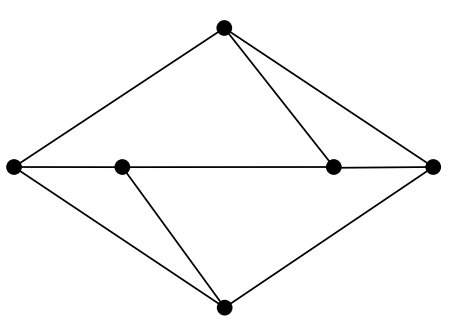

(a) Triangular prism

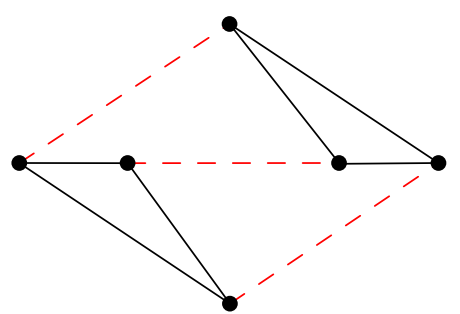

(b) Elongated triangular prism

Figure 3: Triangular prism and elongated triangular prism

prism if it is a triangular prism or if it is obtained by some sequence of subdivisions of the red dashed edges of the triangular prism depicted in Figure 3b.

An outerplanar drawing is a drawing of a graph on a disk in which no two edges cross and all the vertices of the graph are located on the boundary of the disk. A graph is outerplanar if it has an outerplanar drawing.

We also characterise non-separating planar graphs in terms of their structure as follows.

Theorem 2. Any non-separating planar graph is one of the following:

1. an outerplanar graph,

2. a subgraph of a wheel,

3. a subgraph of an elongated triangular prism.

A realisation $\mathcal{R}$ of a graph $G=(V, E)$ in $\mathbb{R}^{3}$ consists of a set of points in $\mathbb{R}^{3}$ that represent (under a bijection) the vertices of the graph and a set of curves between certain pairs of points that represent the edges between corresponding vertex pairs of the graph, such that these curves do not intersect and also do not pass through the points that represent the vertices of the graph. Informally, realisations of graphs are drawings of graphs in $\mathbb{R}^{3}$.

Two vertex-disjoint cycles $C_{1}$ and $C_{2}$ that are embedded into $\mathbb{R}^{3}$ are linked if no topological sphere can be embedded into $\mathbb{R}^{3}$ separating $C_{1}$ from $C_{2}$. Two linked cycles are called a link. To put it in another way, two cycles $C_{1}$ and $C_{2}$ are not linked (unlinked) if they can be continuously deformed without ever intersecting each other until $C_{1}$ and $C_{2}$ end up in two different sides of a topological sphere embedded into $\mathbb{R}^{3}$. Informally, a link consists of two cycles that are embedded in three dimensions such that they cannot be separated unless we cut one of them.

A realisation $\mathcal{R}$ of a graph is linkless if it contains no links. A graph is linklessly embeddable if it has a linkless realisation. Although linklessly embeddable graphs are characterised in terms of a set of forbidden minors proved by Robertson, Seymour, and Thomas [14], there are a lot of unanswered questions about them.

In 1983, Sachs asked for the maximum number of edges in a linklessly embeddable graph on $n$ vertices [15, Problem 2]. Sachs observed that for $n \geqslant 4$, there exists a linklessly embeddable graph with $n$ vertices and $4 n-10$ edges, obtained from a maximal planar 
graph by adding a vertex adjacent to all other vertices [15]. Since a linklessly embeddable graph does not contain a $K_{6}$-minor [15], it follows from a theorem of Mader [9] that a linklessly embeddable graph on $n \geqslant 4$ vertices has at most $4 n-10$ edges. This answers the question of Sachs [15].

Every maximal planar graph on $n$ vertices has the maximum number of edges in an $n$ vertex planar graph. Does the same phenomenon hold for linklessly embeddable graphs? We provide a negative answer by presenting maximal linklessly embeddable graphs on $n$ vertices with only $3 n-3$ edges for infinitely many $n$.

Theorem 3. There exists an infinite family $\mathcal{G}$ of maximal linklessly embeddable graphs such that any graph $G \in \mathcal{G}$ has at most $3|V(G)|-3$ edges.

The rest of this paper is organised as follows. Section 2 goes into more details about the different classes of graphs that we are dealing with and describes their relation to each other. Section 3 is dedicated to proving a number of preliminary lemmas that are used later. More specifically, in Section 3 we investigate the structure of the graphs that contain $K_{2,3}$ as a minor but do not contain any of $K_{1} \cup K_{2,3}, K_{1} \cup K_{4}$ or $K_{1,1,3}$ as a minor. These lemmas are structured in this specific manner to be useful in characterising non-separating planar graphs both in terms of forbidden minors and also in terms of their structure. Section 4 then uses the results of Section 3 to prove our main theorems. In Section 5 we demonstrate the relationship of non-separating planar graphs with linklessly embeddable graphs by using Theorem 1 to prove Theorem 3. Lastly, in Section 6, we summarise our results and point out future directions for research.

\section{Background}

The theory of graph minors developed by Robertson and Seymour is one of the most important recent advances in graph theory and combinatorics. This substantial body of work is presented in a series of 23 papers (Graph Minors I-XXIII) over 20 years from 1983 to 2004.

The graph minor theorem (also known as Robertson-Seymour theorem or Wagner's conjecture) can be formulated as follows:

Graph Minor Theorem (Robertson and Seymour [13]). Every minor-closed class of graphs can be characterised by a finite family of excluded minors.

Perhaps the most famous minor-closed class of graphs is the class of planar graphs. In 1930, Kuratowski characterised planar graphs in terms of two forbidden subdivisions.

Kuratowski's Theorem (Kuratowski [8]). A graph is planar if and only if it does not contain a subdivision of $K_{5}$ or a subdivision of $K_{3,3}$ as a subgraph.

Later on, Wagner characterised planar graphs in terms of forbidden minors as follows:

Wagner's Theorem (Wagner [16]). A graph is planar if and only if it does not have $K_{5}$ or $K_{3,3}$ as a minor. 
In fact, it is easy to see that for any $\operatorname{surface}^{2} \Sigma$, the class of graphs that can be drawn on $\Sigma$ without edge crossings is closed under minors. For example, the class of toroidal graphs is also closed under minors and hence can be characterised in terms of a finite set of forbidden minors. However, the complete set of forbidden minors for this class of graphs is not yet known [10].

Another well-known minor-closed class of graphs is the class of outerplanar graphs. Chartrand and Harary proved that a graph is outerplanar if and only if it does not contain $K_{4}$ or $K_{2,3}$ as a minor [5].

Linklessly embeddable graphs are also closed under minors. Sachs first suggested the study of linklessly embeddable graphs [15]. He conjectured that these embeddings can be characterised by excluding the Petersen family of graphs. The Petersen family of graphs consists of $K_{6}, K_{1,3,3}$ and five other graphs including the Petersen graph.

Conway, Gordon and Sachs proved that $K_{6}$ is not linklessly embeddable [6, 15]. Sachs has also proved that the other members of Petersen family of graphs are not linklessly embeddable [15]. Moreover in the same paper he showed that every minor of a linklessly embeddable graph is linklessly embeddable. Robertson, Seymour and Thomas proved Sach's conjecture in the 1990s [14, 12].

Among the other characterisations of graphs in terms of forbidden minors, we point out the following famous results:

- characterisation of the projective planar graphs (graphs that are embeddable on projective plane) in terms of 35 forbidden minors [2];

- characterisation of outer projective planar graphs (graphs that are embeddable on the projective plane with a disk removed such that all the vertices are located on the boundary of the surface) in terms of 32 forbidden minors [4];

- characterisation of outercylindrical graphs (graphs that are embeddable on a plane with two disks removed from it such that all the vertices are located on the boundary of the removed disks) in terms of 38 forbidden minors [3].

\section{Preliminary Lemmas}

A path $P$ in a graph $G$ is said to be chordless if there is no edge between any two nonconsecutive vertices of $P$ in $G$. A $u v$-path is a path from a vertex $u$ to a vertex $v$. Vertices $u$ and $v$, in a subdivision $S$ of $K_{2,3}$, are called the terminal vertices of $S$ if both $u$ and $v$ have degree 3 in $S$. Define the terminal paths in $S$ as the three $u v$-paths in $S$.

Next we will prove a couple of lemmas about the graphs that do not contain $K_{1,1,3}$ as a minor (see Figure 4).

Lemma 4. Every terminal path in a spanning $K_{2,3}$-subdivision of a $K_{1,1,3}$-minor-free graph is chordless.

\footnotetext{
${ }^{2}$ a 2-manifold, or in other words a topological space such that every point has a neighborhood that is homeomorphic to an open subset of a Euclidean plane
} 


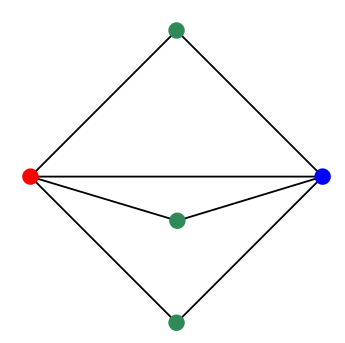

Figure 4: $K_{1,1,3}$

Proof. Suppose that such a terminal path $P$ has a chord $e$. Then it is easy to find a $K_{1,1,3}$ minor in the graph.

A vertex $w$ of a $u v$-path $P$ is an inner vertex of $P$ if $w \neq u$ and $w \neq v$. An edge $e$ of a path $P$ is an inner edge of $P$ if $e$ is incident with two inner vertices of $P$.

Given a set $\mathcal{P}$ of paths in a graph $G$, define a spine $P \in \mathcal{P}$ to be a path such that for any other path $P^{\prime} \in \mathcal{P}$ there is an edge in $G$ that is incident with an inner vertex of $P$ and an inner vertex of $P^{\prime}$. In other words, for each path $P^{\prime} \in \mathcal{P}$ other than $P$ there is an inner vertex of $P$ that is adjacent to an inner vertex of $P^{\prime}$ (see, e.g., Figure 5). Two vertices $u$ and $v$ are co-path with respect to $\mathcal{P}$ if $u$ and $v$ are on the same path in $\mathcal{P}$.

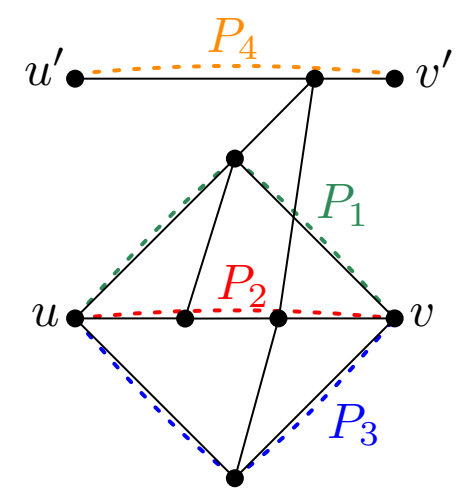

Figure 5: Path $P_{2}$ is the only spine among the four paths $P_{1}, P_{2}, P_{3}, P_{4}$, where $P_{1}, P_{2}, P_{3}$ are $u v$-paths and $P_{4}$ is a $u^{\prime} v^{\prime}$-path (see proof of Lemma 4 ).

Any graph $G$ that contains a $K_{2,3}$-subdivision is spineless if there is no spine among the terminal paths of any of the spanning $K_{2,3}$-subdivisions in $G$. Any graph $G$ with a spanning $K_{2,3}$-subdivision is spineful if it is not spineless.

We divide the rest of lemmas in this section into two subsections, covering spineless and spineful graphs in turn.

\subsection{Spineless Graphs}

We start by proving that spineless graphs do not contain $W_{4}$ as a minor (see Figure 6a).

Lemma 5. If $G$ is a spineless graph then $G$ does not contain $W_{4}$ as a minor. 


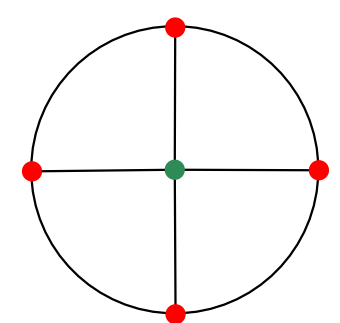

(a) $W_{4}$

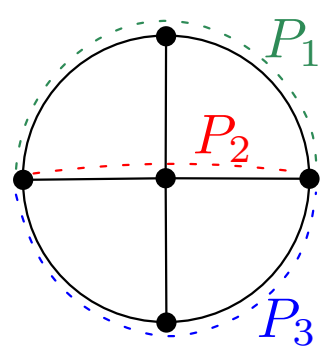

(b) Graph $W_{4}$ with $P_{2}$ as its spine.

Figure 6: Any graph with a spanning $W_{4}$ minor is spineful (see Lemma 5).

Proof. Suppose that there is a spineless graph $G$ that contains $W_{4}$ as a minor. Then it is straightforward to find a $K_{2,3}$-subdivision with a spine in $G$. But this is a contradiction since $G$ is spineless (see, e.g., Figure $6 \mathrm{~b}$ ).

For a subset $U \subseteq V(G), G[U]$ denotes the subgraph of $G$ induced by $U$. Similarly, for any subgraph $H$ of the graph $G, G[H]$ denotes the subgraph of $G$ that is induced by the vertices of $H$.

Lemma 6. Let $P_{1}, P_{2}, P_{3}$ be the terminal paths in a spanning $K_{2,3}$-subdivision $S$ of a spineless graph $G$ with no $K_{1,1,3}$-minor where $G\left[P_{1} \cup P_{2}\right]$ has an edge e that is not in $P_{1}$ or $P_{2}$. Then:

- every edge of $G\left[P_{2} \cup P_{3}\right]$ is an edge of $P_{2} \cup P_{3}$ and every edge of $G\left[P_{1} \cup P_{3}\right]$ is an edge of $P_{1} \cup P_{3}$ and

- $e$ is the only edge in $G\left[P_{1} \cup P_{2}\right]$ that is not in $P_{1}, P_{2}$ and $P_{3}$.

Proof. Let $G_{1}=G\left[P_{1} \cup P_{2}\right], G_{2}=G\left[P_{2} \cup P_{3}\right]$ and $G_{3}=G\left[P_{3} \cup P_{1}\right]$ and let $u$ and $v$ be the two vertices of $e$. First we show that $G_{2}$ does not have any edge that is not an edge of $P_{2}$ or $P_{3}$. To reach a contradiction suppose that $G_{2}$ has an edge $e_{1}=\left(u_{1}, v_{1}\right)$ that is not in $P_{2} \cup P_{3}$. Moreover, by the assumptions of the lemma, there is an edge $e$ in $G_{1}$ that is not in $P_{1} \cup P_{2}$.

By Lemma $4, e$ and $e_{1}$ are not chords of $P_{1}, P_{2}$ or $P_{3}$ and therefore, without loss of generality, $u$ is an inner vertex of $P_{1}$ and $v$ is an inner vertex of $P_{2}$ and $u_{1}$ is an inner vertex of $P_{2}$ and $v_{1}$ is an inner vertex of $P_{3}$ (see, e.g., Figure 7). But this is a contradiction since then $P_{2}$ is a spine and therefore $G$ is not spineless. Similarly we can show that $G_{3}$ does not have any edge that is not an edge of $P_{1}$ or $P_{3}$.

Now we show that there is at most one edge in $G_{1}$ that is not an edge of $P_{1}$ or $P_{2}$. To reach a contradiction suppose that $G_{1}$ has two edges $e_{1}=\left(u_{1}, v_{1}\right)$ and $e_{2}=\left(u_{2}, v_{2}\right)$ that are not among the edges of $P_{1}$ or $P_{2}$ (note that it is possible that either $u_{1}=u_{2}$ or $v_{1}=v_{2}$ or $u_{1}=v_{2}$ or $\left.v_{1}=u_{2}\right)$.

By Lemma $4, e_{1}$ and $e_{2}$ are not chords of $P_{1}$ or $P_{2}$ and therefore, without loss of generality, let $u_{1}$ and $u_{2}$ be among the inner vertices of $P_{1}$ and $v_{1}$ and $v_{2}$ be among the inner vertices of $P_{2}$ (see, e.g., Figure 8). 


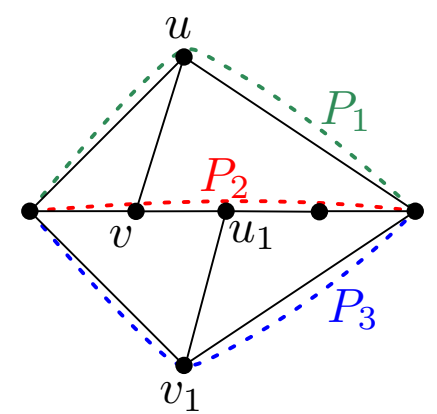

Figure 7: Vertices $u, v, u_{1}, v_{1}$ in $G$ (see proof of Lemma 6)

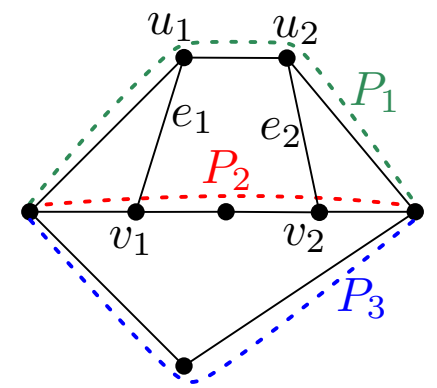

Figure 8: Edges $e_{1}, e_{2}, P_{1}, P_{2}$ and $P_{3}$ in $G$ (see proof of Lemma 6)

Choose $P$ to be either $P_{1}$ or $P_{2}$ so that the endpoints of $e_{1}$ and $e_{2}$ on the other path are distinct. Let $G^{-}$be the graph that is obtained by contracting all the edges of $P$ except the ones that are incident to the terminal vertices of $S$ into a single vertex $w$. It is easy to see that there is a $W_{4}$-minor in $G^{-}$(see, e.g., Figure 9 ). Then by Lemma $5, G$ is not spineless, which is a contradiction.

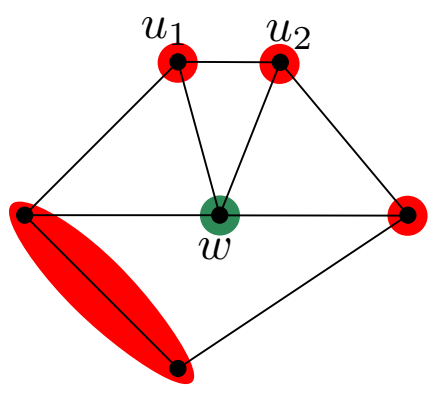

(a) $G^{-}$

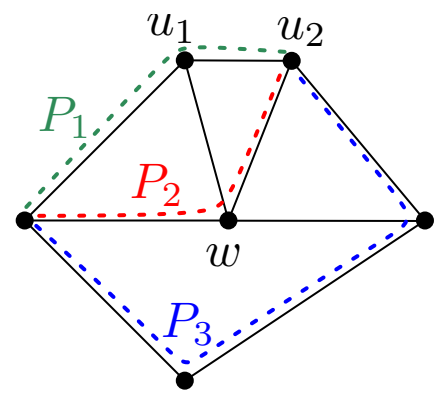

(b) Graph $G^{-}$with a spine $P_{2}$

Figure 9: Finding a spine in $G^{-}$(see proof of Lemma 6)

Lemma 7. Let $\{u, v\}$ and $\left\{P_{1}, P_{2}, P_{3}\right\}$ be the sets of terminal vertices and terminal paths respectively in a spanning $K_{2,3}$-subdivision $S$ of a spineless graph $G$ with no $K_{1,1,3}$-minor and no $\left(K_{1} \cup K_{2,3}\right)$-minor where the lengths of $P_{1}$ and $P_{2}$ are greater than 2 and $G\left[P_{1} \cup P_{2}\right]$ has an edge $e^{\prime}=\left(u^{\prime}, v^{\prime}\right)$ that is not in $P_{1} \cup P_{2}$. Then either: 
- $u^{\prime}$ and $v^{\prime}$ are adjacent to $u$, or

- $u^{\prime}$ and $v^{\prime}$ are adjacent to $v$.

Proof. By Lemma 4, $e^{\prime}$ is not a chord of $P_{1}$ or $P_{2}$ and therefore, without loss of generality, let $u^{\prime}$ be an inner vertex of $P_{1}$ and $v^{\prime}$ be an inner vertex of $P_{2}$. To reach a contradiction, suppose that $u^{\prime}$ and $v^{\prime}$ are not both adjacent to the same vertex $u$ or $v$. We have two cases:

Case 1. Neither $\boldsymbol{u}^{\prime}$ nor $\boldsymbol{v}^{\prime}$ is adjacent to the terminal vertices. In this case it is easy to find a $K_{1} \cup K_{2,3}$ minor in $G$ (see, e.g., Figure 10 and Figure 18a).

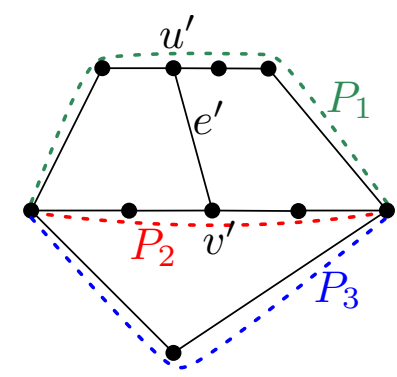

(a) Edge $e^{\prime}$ and $P_{1}, P_{2}, P_{3}$ in $G$

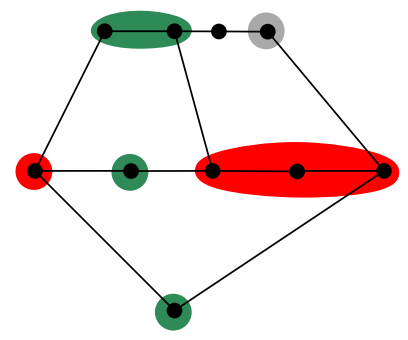

(b) Paths $P_{1}^{\prime}, P_{1}^{\prime \prime}$ in $G$

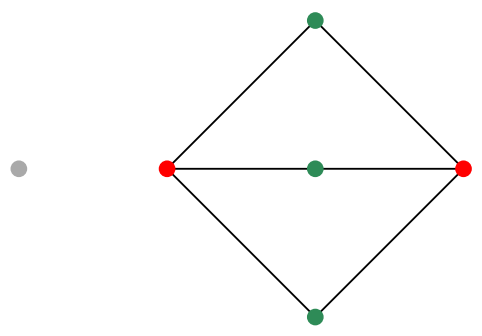

(c) $K_{1} \cup K_{2,3}$

Figure 10: Edge $e^{\prime}$ and $P_{1}, P_{2}, P_{3}, P_{1}^{\prime}, P_{1}^{\prime \prime}$ in $G$. Compare the colouring scheme of Figure $10 \mathrm{~b}$ with Figure 18 a to see how $K_{1} \cup K_{2,3}$ is a minor of $G$ (see proof of Lemma 7 ).

Case 2. One of the two vertices $\boldsymbol{u}^{\prime}$ or $\boldsymbol{v}^{\prime}$ is adjacent to $\boldsymbol{u}$ or $\boldsymbol{v}$. Without loss of generality let $u^{\prime}$ be adjacent to $u$ (see, e.g., Figure 11a).

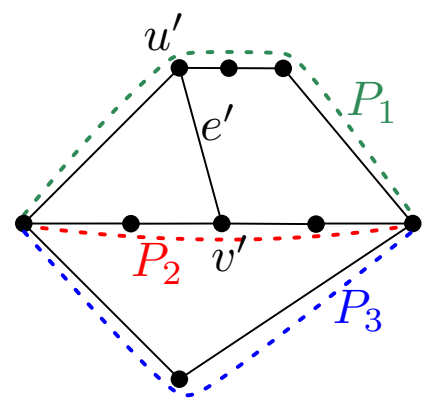

(a) Edge $e^{\prime}$ and $P_{1}, P_{2}, P_{3}$ in $G$

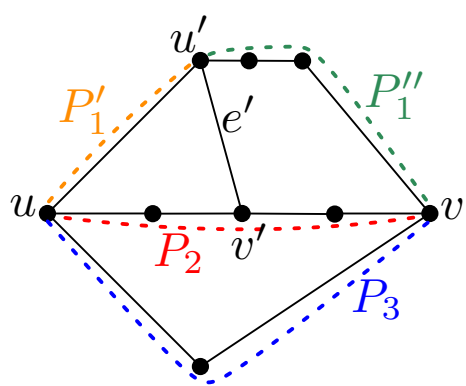

(b) Paths $P_{1}^{\prime}$ and $P_{1}^{\prime \prime}$ in $G$

Figure 11: Edge $e^{\prime}$ and paths $P_{1}, P_{2}, P_{3}, P_{1}^{\prime}, P_{1}^{\prime \prime}$ in $G$ (see proof of Lemma 7).

The vertex $u^{\prime}$ splits $P_{1}$ into two shorter paths $P_{1}^{\prime}$ and $P_{1}^{\prime \prime}$, where $P_{1}$ consists of the edge $\left(u, u^{\prime}\right)$. Without loss of generality, let $P_{1}^{\prime}$ be a shortest path among $P_{1}^{\prime}$ and $P_{1}^{\prime \prime}$ (see, e.g., Figure 11b). Then, since the lengths of $P_{1}$ and $P_{2}$ are greater than 2 , it is easy to see that there is a $K_{2,3}$ minor in $P_{1}^{\prime} \cup e^{\prime} \cup P_{2} \cup P_{3}$ and an inner vertex $v^{\prime \prime}$ on $P_{1}^{\prime \prime}$ such that $P_{1}^{\prime} \cup e^{\prime} \cup P_{2} \cup P_{3}$ and $v^{\prime \prime}$ form a $K_{1} \cup K_{2,3}$ minor in $G$ (see, e.g., Figure 12). However, this is a contradiction since $G$ is a $K_{1} \cup K_{2,3}$-minor free graph. 


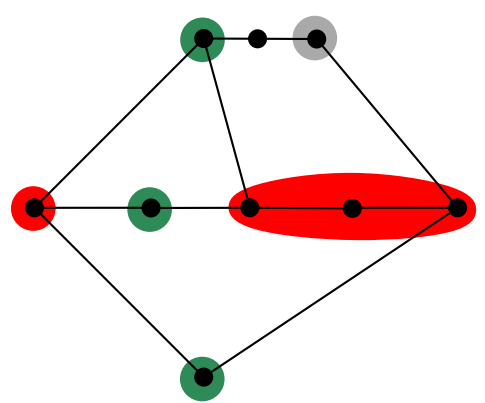

Figure 12: Finding a $K_{1} \cup K_{2,3}$-minor in $G$ (compare with Figure 10c, see proof of Lemma 7).

Lemma 8. Let $\mathcal{G}$ be the family of spineless graphs with no $K_{1,1,3}$-minor and no $\left(K_{1} \cup\right.$ $\left.K_{2,3}\right)$-minor, and that contain a $K_{2,3}$-subdivision. Then any $G \in \mathcal{G}$ can be obtained by subdividing the red dashed edges of the graphs that are shown in Figure 13.

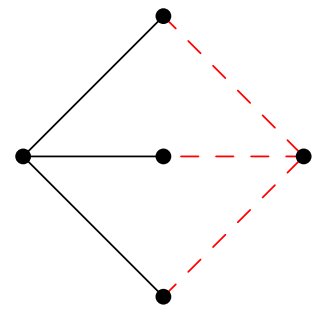

(a) Type I

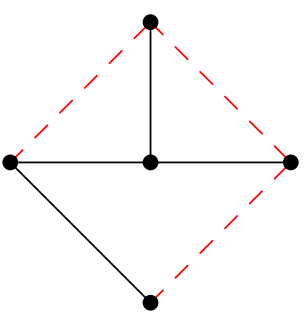

(b) Type II

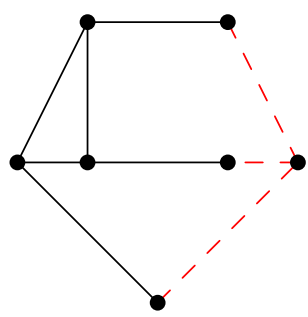

(c) Type III

Figure 13: Three types of spineless non-separating planar graphs (see proof of Lemma 8).

Proof. Let $P_{1}, P_{2}, P_{3}$ be the terminal paths and $u, v$ be the terminal vertices in a $K_{2,3^{-}}$ subdivision $S$ of a graph $G \in \mathcal{G}$. Since $G$ does not contain $K_{1} \cup K_{2,3}$ as a minor, $S$ is a spanning $K_{2,3}$-subdivision of $G$. If $G$ does not have any edges other than the edges of $P_{1}, P_{2}, P_{3}$ then, clearly, $G$ can be obtained by subdividing the red dashed edges of the graph depicted in Figure 13a.

Now let us consider the case where $G$ has an edge $e^{\prime}=\left(u^{\prime}, v^{\prime}\right)$ that is not an edge of any of $P_{1}, P_{2}, P_{3}$. By Lemma $6, e^{\prime}$ is the only edge in $G$ that is not an edge of $P_{1}, P_{2}$ or $P_{3}$. By Lemma $4, e^{\prime}$ is not a chord of $P_{1}, P_{2}$ or $P_{3}$ and therefore, without loss of generality, let $u^{\prime}$ be an inner vertex of $P_{1}$ and $v^{\prime}$ be an inner vertex of $P_{2}$. We have two cases:

Case 1. Either $\boldsymbol{P}_{\mathbf{1}}$ or $\boldsymbol{P}_{\mathbf{2}}$ has length 2 . It is easy to verify that in this case $G$ is a graph that can be obtained by subdividing the red dashed edges in Figure 13b.

Case 2. The lengths of both $\boldsymbol{P}_{1}$ and $\boldsymbol{P}_{\mathbf{2}}$ are more than 2. By Lemma 7 , both $u^{\prime}$ and $v^{\prime}$ are adjacent to the same vertex $u$ or $v$. Now it is easy to verify that in this case $G$ is a graph that can be obtained by subdividing the red dashed edges in Figure 13c. 


\subsection{Spineful Graphs}

Lemma 9. There is at most one spine in the set of terminal paths of a spanning $K_{2,3^{-}}$ subdivision of a $K_{1,1,3}$-minor-free graph.

Proof. Let $\mathcal{P}=\left\{P_{1}, P_{2}, P_{3}\right\}$ be the set of terminal paths in a spanning $K_{2,3}$-subdivision $S$ in a $K_{1,1,3}$-minor-free graph $G$. To reach a contradiction, suppose that there is more than one spine in $\mathcal{P}$. Without loss of generality, let $P_{1}$ and $P_{2}$ both be spines. Then, for each pair $i, j \in\{1,2,3\}$ with $i<j$, there is an edge incident with an inner vertex of $P_{i}$ and an inner vertex of $P_{j}$. Now, it is easy to find $K_{1,1,3}$ as a minor in $G$. See Figure 14.

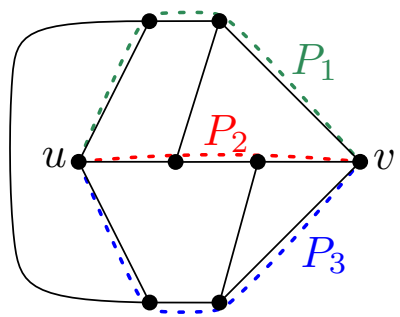

(a) Graph $G$ with $P_{1}$ and $P_{2}$ as spines.

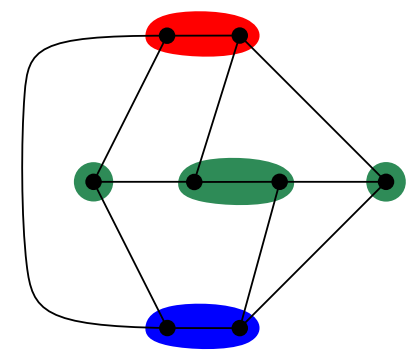

(b) Graph $G$ contains $K_{1,1,3}$ as a minor.

Figure 14: If $P_{1}$ and $P_{2}$ are spines then $G$ contains $K_{1,1,3}$ as a minor. The colour scheme used here to colour the vertices of a $K_{1,1,3}$ minor is the same as the one used in Figure 4 (see proof of Lemma 9).

Next we will prove a lemma about a class of graphs that do not contain $K_{1} \cup K_{4}$ as a minor (see Figure 15).

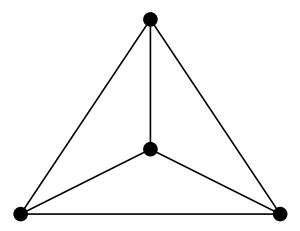

Figure 15: $K_{1} \cup K_{4}$

Lemma 10. Let $P_{1}, P_{2}, P_{3}$ be the terminal paths in a spanning $K_{2,3}$-subdivision $S$ of a graph $G$ with no $\left(K_{1} \cup K_{4}\right)$-minor, where $P_{2}$ is a spine. Then there is no pair of edges $e_{1}=\left(u_{1}, v_{1}\right)$ and $e_{2}=\left(u_{1}, v_{2}\right)$ in $G$ such that $u_{1}$ is an inner vertex of $P_{1}$ or $P_{3}$ and $v_{1}$ and $v_{2}$ are two distinct inner vertices of $P_{2}$.

Proof. To reach a contradiction suppose that there is an edge $e_{1}=\left(u_{1}, v_{1}\right)$ and an edge $e_{2}=\left(u_{1}, v_{2}\right)$ such that $u_{1}$ is an inner vertex of $P_{1}$ or $P_{3}$ and $v_{1}$ and $v_{2}$ are two inner vertices of $P_{2}$ (see, e.g., Figure 16a). Without loss of generality let $u_{1}$ be an inner vertex of $P_{1}$. Since $P_{2}$ is a spine there is also an edge $e_{3}=\left(u_{3}, v_{3}\right)$ in $G$ such that $u_{3}$ is an inner 


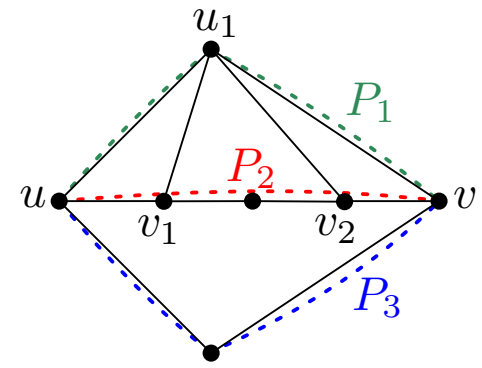

(a) Graph $G$ with $P_{2}$ as a spine.

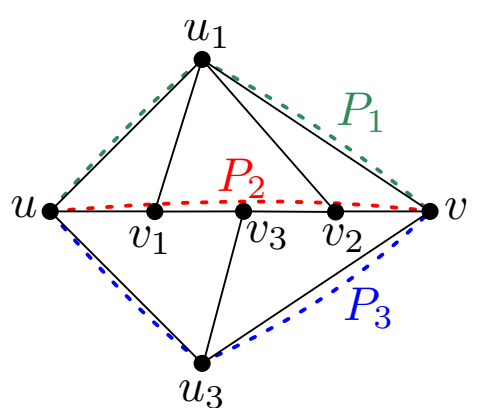

(b) Graph $G$ contains $K_{1,1,3}$ as a minor.

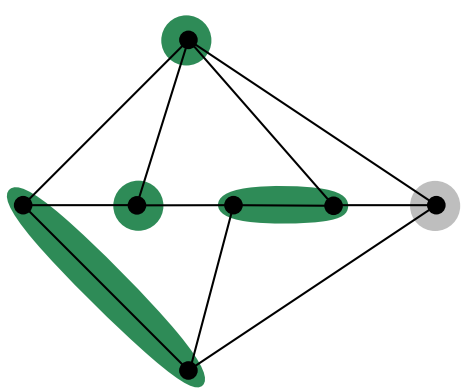

(c) Finding a $K_{1} \cup K_{4}$ minor in $G$.

Figure 16: Graph $G$ contains $K_{1} \cup K_{4}$ as a minor (see proof of Lemma 10).

vertex of $P_{3}$ and $v_{3}$ is an inner vertex of $P_{2}$ (see, e.g., Figure 16b). Now it is easy to find a $\left(K_{1} \cup K_{4}\right)$-minor in $G$ (see, e.g., Figure 16c).

Let $P$ be a path and $h$ be a vertex that is not in $P$. Let $G$ be the graph that is obtained from $P$ and $h$ by adding an edge $(h, v)$ for every vertex $v$ in $P$. Then $G$ is a fan graph and $h$ is the handle of $G .^{3}$

Let $P$ be a $u v$-path. We define the outer inner vertices of $P$ as those inner vertices of $P$ that are adjacent to $u$ and $v$ on $P$.

Lemma 11. Let $P_{1}, P_{2}, P_{3}$ be the terminal paths in a spanning $K_{2,3}$-subdivision $S$ of a graph $G$ with no $K_{1,1,3}$-minor, no $\left(K_{1} \cup K_{4}\right)$-minor and no $\left(K_{1} \cup K_{2,3}\right)$-minor, where $P_{2}$ is a spine. Then, $G\left[P_{1} \cup P_{2}\right]$ and $G\left[P_{2} \cup P_{3}\right]$ are subgraphs of fan graphs whose handles are among the outer inner vertices of $P_{2}$.

Proof. Let $G_{1}=G\left[P_{1} \cup P_{2}\right]$ and $G_{2}=G\left[P_{2} \cup P_{3}\right]$. First we show that $G_{1}$ and $G_{2}$ are subgraphs of fan graphs. To reach a contradiction suppose that either $G_{1}$ or $G_{2}$ is not a subgraph of a fan graph. Without loss of generality, suppose that $G_{1}$ is not a subgraph of a fan graph.

Since $G_{1}$ is not a subgraph of a fan graph, there are two edges $e_{1}=\left(u_{1}, v_{1}\right)$ and $e_{2}=\left(u_{2}, v_{2}\right)$ in $G_{1}$ that are not an edge of $P_{1}$ or an edge of $P_{2}$ and are vertex-disjoint. By Lemma $4, e_{1}$ and $e_{2}$ are not chords of $P_{1}$ or $P_{2}$. In other words:

- $u_{1}, v_{1}, u_{2}, v_{2}$ are all inner vertices of $P_{1}$ and $P_{2}$.

- $u_{1}$ and $v_{1}$ are not co-path with respect to $\left\{P_{1}, P_{2}, P_{3}\right\}$.

- $u_{2}$ and $v_{2}$ are not co-path with respect to $\left\{P_{1}, P_{2}, P_{3}\right\}$.

Without loss of generality let $u_{1}$ and $u_{2}$ be the two endpoints of $e_{1}$ and $e_{2}$ on $P_{1}$ and let $v_{1}$ and $v_{2}$ be the other two endpoints of $e_{1}$ and $e_{2}$ on $P_{2}$. Let $u$ and $v$ be the terminal

\footnotetext{
${ }^{3} K_{3}$ and $K_{4}$ minus an edge are the only fan graphs that do not have a unique handle.
} 
vertices of $S$. Contract all the edges of $P_{1}$ that are not incident to $u$ and $v$ into a single vertex $w$ and let us denote the resulting minor of $G$ by $H$.

Since $H$ is a minor of $G$, it does not contain a $K_{1} \cup K_{4}$ minor. Moreover, $P_{2}$ is a spine in $H$. Also, $w$ is adjacent to $v_{1}$ and $v_{2}$ in $H$. Therefore, $e_{1}=\left(w, v_{1}\right)$ and $e_{2}=\left(w, v_{2}\right)$ are two edges of $H$ that contradict Lemma 10 and therefore $G_{1}$ is a subgraph of a fan graph. We denote the corresponding fan graph by $G_{1}^{+}$.

Similarly, we conclude that $G_{2}$ is a subgraph of a fan graph and we denote the corresponding fan graph by $G_{2}^{+}$.

Next we show that the handles of fan graphs $G_{1}^{+}$and $G_{2}^{+}$, which we denote by $h_{1}$ and $h_{2}$ respectively, are outer inner vertices of $P_{2}$. As the first step, we show that $h_{1}$ and $h_{2}$ are inner vertices of $P_{2}$ and then as the second step we show that both $h_{1}$ and $h_{2}$ are adjacent to either $u$ or $v$ on $P_{2}$ (i.e., $h_{1}$ and $h_{2}$ are outer inner vertices of $P_{2}$ ).

We use contradiction to prove the first step. To reach a contradiction suppose that either the handle of $G_{1}^{+}$or the handle of $G_{2}^{+}$is not an inner vertex of $P_{2}$. Without loss of generality, suppose that the handle of $G_{1}^{+}$is not an inner vertex of $P_{2}$. Then it must be on $P_{1}$. So there are two edges $e_{1}=\left(u_{1}^{\prime}, v^{\prime}\right)$ and $e_{2}=\left(u_{2}^{\prime}, v^{\prime}\right)$ in $G_{1}$ that are not in $E\left(P_{1}\right) \cup E\left(P_{2}\right)$ and are incident with the same vertex $v^{\prime}$ on $P_{1}$.

By Lemma $4, e_{1}$ and $e_{2}$ are not chords of $P_{1}$ or $P_{2}$ and therefore $v^{\prime}$ is an inner vertex of $P_{1}$ and $u_{1}^{\prime}$ and $u_{2}^{\prime}$ are inner vertices of $P_{2}$. However, this is also in contradiction with Lemma 10.

We use contradiction to prove the second step as well. To reach a contradiction, without loss of generality, suppose that $h_{1}$ is not adjacent to $u$ or $v$ on $P_{2}$ and let $h_{2}$ be any vertex on $P_{2}$. The handle $h_{2}$ splits $P_{2}$ into two subpaths: $P_{2}^{\prime}$ from $u$ to $h_{2}$ and $P_{2}^{\prime \prime}$ from $h_{2}$ to $v$. Without loss of generality, let $h_{1}$ be on $P_{2}^{\prime}$ (see, e.g., Figure 17a).

Since $P_{2}$ is a spine, there are two edges $e_{1}=\left(u_{1}, x_{1}\right)$ and $e_{2}=\left(u_{2}, x_{2}\right)$ such that $u_{1}$ is an inner vertex of $P_{1}$ and $u_{2}$ is an inner vertex of $P_{3}$. Since $G_{1}$ is a subgraph of a fan graph $G_{1}^{+}$with handle $h_{1}$ we have $x_{1}=h_{1}$ and since $G_{2}$ is a subgraph of fan graph $G_{2}^{+}$ with handle $h_{2}$ we have $x_{2}=h_{2}$ (see, e.g., Figure 17b). Let $P_{1}^{\prime}$ be the part of $P_{1}$ from $u$ to $u_{1}$ and let $P_{3}^{\prime}$ be the part of $P_{3}$ from $u$ to $u_{2}$.

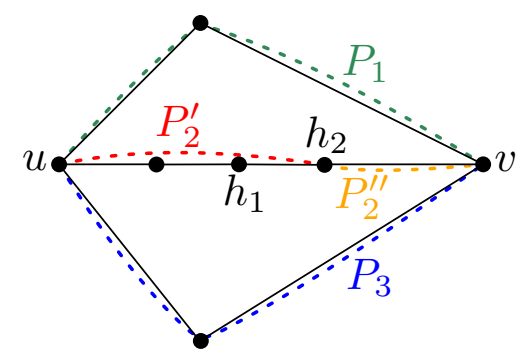

(a) Vertices $h_{1}, h_{2}$ and $P_{2}^{\prime}, P_{2}^{\prime \prime}$ in $G$.

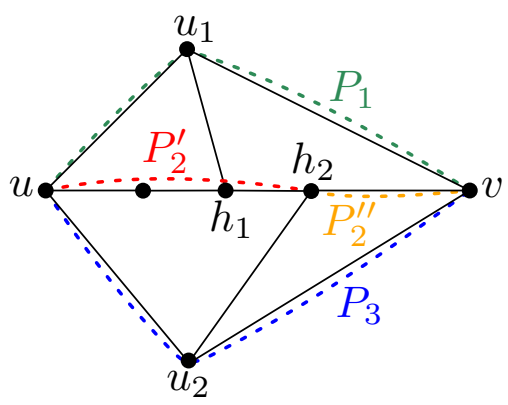

(b) Vertices $u_{1}, u_{2}$ in $G$.

Figure 17: Finding $K_{1} \cup K_{2,3}$ minor in $G$ (see proof of Lemma 11).

Now it is easy to see that $v$ together with $P_{1}^{\prime} \cup\left(u_{1}, h_{1}\right) \cup P_{2}^{\prime} \cup\left(u_{2}, h_{2}\right) \cup P_{3}^{\prime}$ contains a 
$K_{1} \cup K_{2,3}$ minor, which is a contradiction (see, e.g., Figure 18).

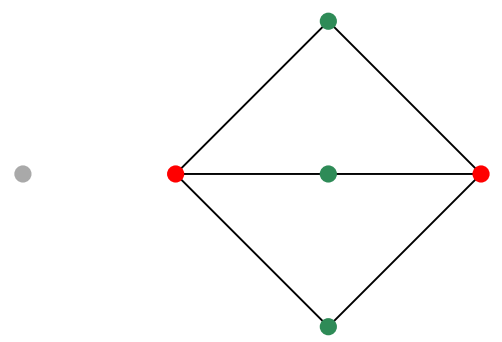

(a) $K_{1} \cup K_{2,3}$

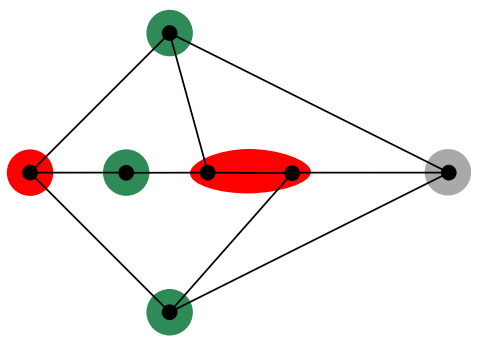

(b) Graph $G$ contains $K_{1} \cup K_{2,3}$ as a minor.

Figure 18: Finding $K_{1} \cup K_{2,3}$ minor in $G$ (see proof of Lemma 11).

Lemma 12. Let $G$ be a $K_{1,1,3}$-minor-free, $\left(K_{1} \cup K_{4}\right)$-minor-free, $\left(K_{1} \cup K_{2,3}\right)$-minor-free graph with a spanning $K_{2,3}$-subdivision $S$. Let $G\left[P_{1} \cup P_{2}\right]$ and $G\left[P_{2} \cup P_{3}\right]$ be subgraphs of fan graphs $G_{1}^{+}$and $G_{2}^{+}$with the same handle $h$ where $P_{1}, P_{2}, P_{3}$ are the terminal paths in $S$ and $P_{2}$ is a spine. Then the length of $P_{2}$ is 2.

Proof. To reach a contradiction suppose that length of $P_{2}$ is greater than 2. Since $P_{2}$ is the spine, by Lemma 11, $h$ is an outer inner vertex of $P_{2}$. Now, it is easy to find a $K_{1} \cup K_{2,3}$ minor in $G$ which contradicts the assumptions of the lemma (see, e.g., Figure 19).

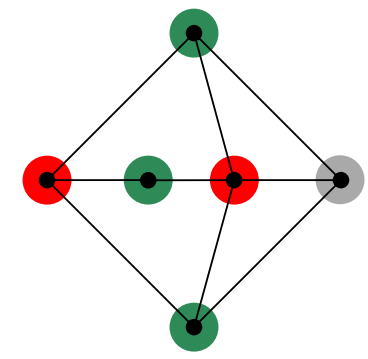

Figure 19: Finding a $\left(K_{1} \cup K_{2,3}\right)$-minor in $G$ (see proof of Lemma 12).

Lemma 13. Let $G$ be a $K_{1,1,3}$-minor-free, $\left(K_{1} \cup K_{4}\right)$-minor-free, $\left(K_{1} \cup K_{2,3}\right)$-minor-free graph with a spanning $K_{2,3}$-subdivision $S$. Let $u, v$ be the terminal vertices and $P_{1}, P_{2}, P_{3}$ be the terminal paths in $S$ and let $P_{2}$ be a spine in $S$. Let $G_{1}=G\left[P_{1} \cup P_{2}\right]$ and $G_{2}=G\left[P_{2} \cup P_{3}\right]$ be subgraphs of fan graphs $G_{1}^{+}$with handle $h_{1}$ and $G_{2}^{+}$with handle $h_{2}$ respectively such that $h_{1} \neq h_{2}$.

Then there is exactly one edge $e^{\prime}=\left(h_{1}, v^{\prime}\right)$ in $G_{1}$ that is not in $P_{1} \cup P_{2}$ and there is exactly one edge $e^{\prime \prime}=\left(h_{2}, v^{\prime \prime}\right)$ in $G_{2}$ that is not in $P_{2} \cup P_{3}$, where:

- $h_{1}$ and $v^{\prime}$ are outer inner vertices of $P_{2}$ and $P_{1}$ respectively that are both adjacent to $u$ or both adjacent to $v$ and 
- $h_{2}$ and $v^{\prime \prime}$ are outer inner vertices of $P_{2}$ and $P_{3}$ respectively that are both adjacent to $u$ or both adjacent to $v$.

Proof. Since $P_{2}$ is a spine, there is an edge $e^{\prime}=\left(h_{1}, v^{\prime}\right)$ in $G_{1}$ that is not in $P_{1} \cup P_{2}$ and there is an edge $e^{\prime \prime}=\left(h_{2}, v^{\prime \prime}\right)$ in $G_{2}$ that is not in $P_{2} \cup P_{3}$. Moreover, by Lemma $11, h_{1}$ and $h_{2}$ are outer inner edges of $P_{2}$.

Now to reach a contradiction, without loss of generality, let $h_{1}$ be adjacent to $u$ on $P_{2}$ but let $v^{\prime}$ be a vertex that is not adjacent to $u$ on $P_{1}$. Let $v_{1}$ be the vertex that is adjacent to $u$ on $P_{1}$.

Since $h_{1}$ and $h_{2}$ are inner vertices of the spine $P_{2}$, by Lemma $4, v^{\prime}$ is an inner vertex of $P_{1}$ and $v^{\prime \prime}$ is an inner vertex of $P_{2}$ (see, e.g., Figure 20a).

We know that $v_{1}$ appears before $v^{\prime}$ as we traverse $P_{1}$ from $u$ towards $v$ and $h_{1}$ appears before $h_{2}$ as we traverse $P_{2}$ from $u$ towards $v$. Let $P^{\prime}$ be the part of $P_{1}$ that stretches from $v^{\prime}$ to $v$. Now it is easy to see that $v_{1}$ together with $\left(h_{1}, v^{\prime}\right) \cup P^{\prime} \cup P_{2} \cup P_{3}$ contains a $K_{1} \cup K_{2,3}$ minor, which is a contradiction (see, e.g., Figure 20b).

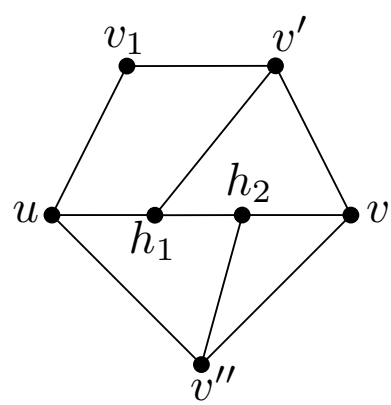

(a) $K_{1} \cup K_{2,3}$

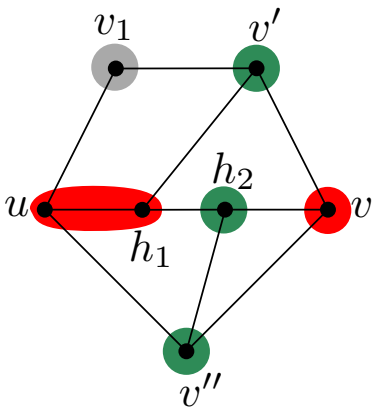

(b) Graph $G$ contains $K_{1} \cup$ $K_{2,3}$ as a minor.

Figure 20: Finding a $\left(K_{1} \cup K_{2,3}\right)$-minor in $G$ (see proof of Lemma 13).

Lemma 14. Let $\mathcal{G}$ be the family of spineful $K_{1,1,3}$-minor-free, $\left(K_{1} \cup K_{4}\right)$-minor-free and $\left(K_{1} \cup K_{2,3}\right)$-minor-free graphs that contain a $K_{2,3}$-subdivision. Then for any $G \in \mathcal{G}$, either $G$ is a subgraph of a wheel with at least 4 spokes or $G$ is an elongated triangular prism.

Proof. Let $P_{1}, P_{2}, P_{3}$ be the terminal paths and $u, v$ be the terminal vertices in a $K_{2,3^{-}}$ subdivision $S$ of a graph $G \in \mathcal{G}$ where $P_{2}$ is a spine. Since $G$ does not contain $K_{1} \cup K_{2,3}$ as a minor, $S$ is a spanning $K_{2,3}$-subdivision of $G$. Let $G_{1}=G\left[P_{1} \cup P_{2}\right]$ and $G_{2}=G\left[P_{2} \cup P_{3}\right]$. Since $P_{2}$ is a spine, by Lemma 11, $G_{1}$ and $G_{2}$ are subgraphs of fan graph $G_{1}^{+}$and $G_{2}^{+}$with handles $h_{1}$ and $h_{2}$ where $h_{1}$ and $h_{2}$ are both among the outer inner vertices of $P_{2}$.

We break the rest of the proof into two cases:

Case $\mathbf{1} \cdot \boldsymbol{h}_{\mathbf{1}}=\boldsymbol{h}_{\mathbf{2}}$. By Lemma 12, the length of $P_{2}$ is 2 and therefore $G$ is a subgraph of a wheel $W$. Moreover, since $P_{2}$ is a spine, $W$ has at least 4 spokes.

Case 2. $\boldsymbol{h}_{\mathbf{1}} \neq \boldsymbol{h}_{\mathbf{2}}$. By Lemma 13, there is exactly one edge $e_{1}$ in $G_{1}$ that is not in $P_{1} \cup P_{2}$ and exactly one edge $e_{2}$ in $G_{2}$ that is not in $P_{2} \cup P_{3}$. Then $G$ is an elongated triangular prism. 


\section{Proof of the Main Theorems}

Lemma 15. A graph $G$ does not contain any of $K_{1} \cup K_{4}$ or $K_{1} \cup K_{2,3}$ or $K_{1,1,3}$ as a minor if and only if $G$ is either an outerplanar graph or a subgraph of a wheel or a subgraph of an elongated triangular prism.

Proof. It is straightforward to see that any outerplanar graph or a subgraph of a wheel or an elongated triangular prism does not contain any of $K_{1} \cup K_{4}$ or $K_{1} \cup K_{2,3}$ or $K_{1,1,3}$ as a minor. Next we prove the lemma in the other direction.

We break the proof into the following three cases:

1. $G$ does not contain any of $K_{4}$ or $K_{2,3}$ as a minor.

2. $G$ contains $K_{4}$ but does not contain $K_{2,3}$ as a minor.

3. $G$ contains $K_{2,3}$ as a minor.

Case 1. Graph $G$ does not contain any of $K_{4}$ or $K_{2,3}$ as a minor. In this case, $G$ is outerplanar.

Case 2. Graph $G$ contains $K_{4}$ as a minor but it does not contain $K_{2,3}$ as a minor. Since the degrees of the vertices in $K_{4}$ are less than 4 , any subgraph contractible to $K_{4}$ is also a subdivision of $K_{4}$. Therefore, there is a subdivision $S$ of $K_{4}$ in $G$.

Since $G$ does not contain $K_{1} \cup K_{4}$ as a minor, $S$ is a spanning subgraph of $G$ (any vertex of $G$ is also a vertex of $S$ ). Moreover, since any proper subdivision of $K_{4}$ contains $K_{2,3}$ as a minor, $K_{4}$ is the only graph that contains $K_{4}$ as a minor but does not contain $K_{2,3}$ as a minor. So $G$ is isomorphic to $K_{4}$ and is a subgraph of a wheel.

Case 3. Graph $G$ contains $K_{2,3}$ as a minor. Since the degrees of the vertices in $K_{2,3}$ are less than 4 , any subgraph contractible to $K_{2,3}$ is also a subdivision of $K_{2,3}$. Therefore, there is a subdivision $S$ of $K_{2,3}$ in $G$. Since $G$ does not contain $K_{1} \cup K_{2,3}$ as a minor, $S$ is a spanning subgraph of $G$.

Here we have two cases:

Case 3a. Graph $\boldsymbol{G}$ is spineless. By Lemma 8, $G$ can be obtained by subdividing the red dashed edges of one of the graphs shown in Figure 13. Now, any of the graphs shown in Figure 13 is a subgraph of a wheel or a subgraph of an elongated triangular prism. Therefore $G$ is either a subgraph of a wheel or a subgraph of an elongated triangular prism.

Case 3b. Graph $\boldsymbol{G}$ is spineful. By Lemma 14, $G$ is either a subgraph of a wheel or it is a subgraph of an elongated triangular prism.

\subsection{Proof of Theorem 1}

Lemma 16. For any graph $G, G$ contains any of $K_{1} \cup K_{4}$ or $K_{1} \cup K_{2,3}$ or $K_{1,1,3}$ as a minor if and only if $G$ contains any of $K_{1} \cup K_{4}$ or $K_{1} \cup K_{2,3}$ or $K_{1,1,3}$ as a subdivision.

Proof. By the definitions of subdivision and minor, if $G$ contains a graph $H$ as a subdivision, then $G$ contains $H$ as a minor. Moreover, if a graph $G$ contains a graph $H$ with 
maximum degree three as a minor, then $G$ contains $H$ as a subdivision. Therefore, as both $K_{1} \cup K_{4}$ and $K_{1} \cup K_{2,3}$ have maximum degree of three, in order to prove this lemma, it is sufficient to show that if $G$ contains $K_{1,1,3}$ as a minor, then $G$ contains $K_{1,1,3}$ or $K_{1} \cup K_{2,3}$ as a subdivision.

Let $G$ be a graph that contains $K_{1,1,3}$ as a minor. Then either $G$ contains $K_{1,1,3}$ as a subdivision and we are done, or $G$ contains a subgraph $H$ that is contractible to the graph $J$ shown in Figure 21a. However, $J$ contains $K_{1} \cup K_{2,3}$ as a subgraph (see Figure 21b). Therefore $G$ has a subgraph contractible to $K_{1} \cup K_{2,3}$. But $K_{1} \cup K_{2,3}$ has maximum degree three and hence $G$ contains a subdivision of $K_{1} \cup K_{2,3}$.

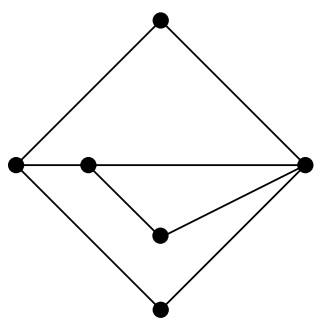

(a) Graph $J$

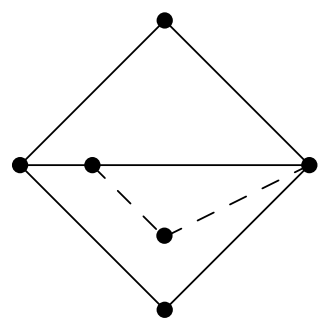

(b) Graph $J$ contains $K_{1} \cup K_{2,3}$ as a subdivision (by deletion of dashed edges).

Figure 21: Finding a $K_{1} \cup K_{2,3}$ subdivision in $J$ (see proof of Lemma 16).

The proof of Lemma 16, in essence, is similar to how we can deduce Kuratowski's characterisation of planar graphs from Wagner's characterisation of planar graphs and vice versa (refer to Kuratowski's Theorem and Wagner's Theorem in Section 2).

Now we are ready to prove Theorem 1 .

Proof of Theorem 1. It is straightforward to verify that in any planar drawing of a graph that contains $K_{1} \cup K_{4}$ or $K_{1} \cup K_{2,3}$ or $K_{1,1,3}$ as a minor, there are two vertices that are separated by a cycle. Moreover, by Lemma 16, graph $G$ contains any of $K_{1} \cup K_{4}$ or $K_{1} \cup K_{2,3}$ or $K_{1,1,3}$ as a minor if and only if $G$ contains any of $K_{1} \cup K_{4}$ or $K_{1} \cup K_{2,3}$ or $K_{1,1,3}$ as a subdivision. Therefore, to prove this theorem, it is sufficient to show that any graph that does not contain any of $K_{1} \cup K_{4}$ or $K_{1} \cup K_{2,3}$ or $K_{1,1,3}$ as a minor is a non-separating planar graph.

By Lemma 15, any graph that does not contain any of $K_{1} \cup K_{4}$ or $K_{1} \cup K_{2,3}$ or $K_{1,1,3}$ as a minor is either an outerplanar graph or a subgraph of a wheel or an elongated triangular prism and it is easy to verify that any such graph is a non-separating planar graph.

\subsection{Proof of Theorem 2}

Proof. Theorem 2 is a direct consequence of Lemma 15 and Theorem 1.

Theorems 1 and 2 together provide us with Theorem 17: 


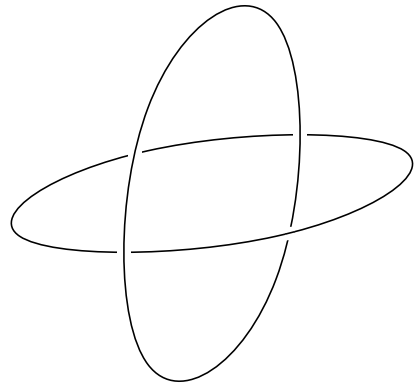

(a) a link in 3D

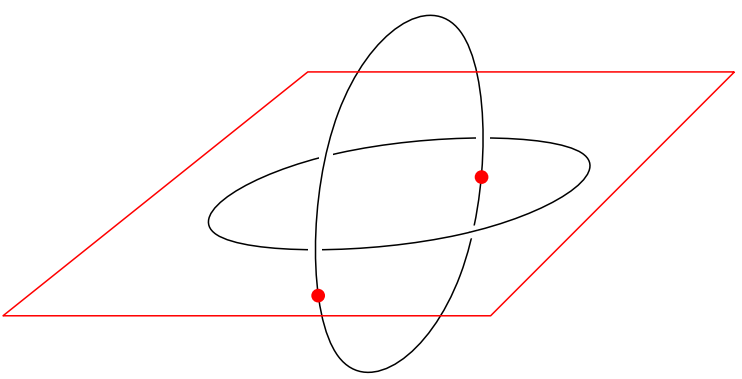

(b) intersection of a plane with a link

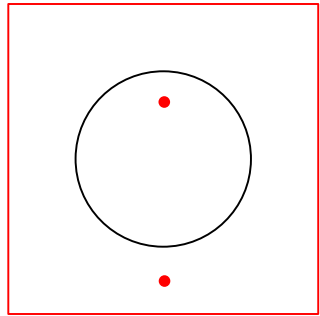

(c) cross section of the link from the top

Figure 22: A separating cycle in a cross section of a link with a plane

Theorem 17. The following are equivalent, for any graph $G$ :

1. $G$ does not contain any of $K_{1} \cup K_{4}$ or $K_{1} \cup K_{2,3}$ or $K_{1,1,3}$ as a minor.

2. $G$ is outerplanar or a subgraph of a wheel or a subgraph of an elongated triangular prism.

3. $G$ is a non-separating planar graph.

\section{$5 \quad$ Proof of Theorem 3}

Consider two linked circles in three dimensions and a cross section of them that contains one of the two circles as depicted in Figure 22a. Such a cross-section has a structure that resembles the structure of a separating cycle with a vertex inside it and another outside it.

With this intuition in mind, we prove Theorem 3.

Proof. Let $G$ be an elongated prism. Then $G$ is a maximal non-separating planar graph with $|V(G)|+3$ edges. Moreover, $G$ contains both $K_{4}$ and $K_{2,3}$ as minors.

Let $H$ be the graph that is obtained by adding two new vertices $u$ and $v$ to $G$ such that $u$ and $v$ are each adjacent to all the vertices of $G$. Graph $H$ has at most $3|V(H)|-3$ edges. We claim that $H$ is a maximal linklessly embeddable graph.

To prove that $H$ is a maximal linklessly embeddable graph, we show that any graph $H^{+}$that is obtained by adding an edge $e$ to $H$ is not a linklessly embeddable graph. Since $u$ and $v$ are adjacent to all the vertices of $G$, edge $e$ (in $H^{+}$) is either $(u, v)$ or it is an edge between two vertices of $G$.

Let $H^{+}$be the graph obtained by adding $(u, v)$ to $H$. Since $G$ contains $K_{4}$ or $K_{2,3}$ as a minor, $H^{+}$contains either $K_{6}$ or $K_{1,1,2,3}$ as a minor. The latter contains $K_{1,3,3}$. However, $K_{6}$ and $K_{1,3,3}$ are both forbidden minors for linklessly embeddable graphs [15, 12].

Now let $H^{+}$be the graph that is obtained by adding an edge between two vertices of $G$ in $H$. By the characterisation of non-separating planar graphs, $G+e$ contains $K_{4} \cup K_{1}$ or $K_{2,3} \cup K_{1}$ or $K_{1,1,3}$ as a minor. 
If $G+e$ contains $K_{4} \cup K_{1}$ as a minor, then $H^{+}$contains $K_{6}$ as a minor and hence $H^{+}$is not a linklessly embeddable graph. If $G+e$ contains $K_{2,3} \cup K_{1}$ as a minor, then $H^{+}$contains $K_{1,1,2,3}$ as a minor. But $K_{1,1,2,3}$ contains $K_{1,3,3}$ as a minor and therefore $\mathrm{H}^{+}$is not a linklessly embeddable graph. If $G+e$ contains $K_{1,1,3}$ as a minor, then $\mathrm{H}^{+}$ contains $K_{2,1,1,3}$ as a minor, which in turn contains $K_{1,3,3}$ as a minor. Therefore $H^{+}$is not a linklessly embeddable graph.

\section{Conclusion}

This paper provides a forbidden minor characterisation for non-separating planar graphs and as expected, the forbidden minors for non-separating planar graphs are each a minor of one of the two forbidden minors for planar graphs.

Moreover it describes the structure of these graphs by proving that any maximal non-separating planar graph is either an outerplanar graph or a wheel or an elongated triangular prism.

One can define a similar class of graphs with respect to surfaces other than the plane. For example, a non-separating toroidal graph is a graph that has a drawing $D$ on the torus such that:

- no two edges cross and

- for any cycle $C$ in $D$ and any two vertices $u$ and $v$ in $D$ that are not a vertex of $C$, one can draw a curve from $u$ to $v$ without crossing any edge of $C$.

Any such class of graphs is also closed under minor operations and hence it can be characterised using a finite set of forbidden minors. It would be specially interesting to know the set of forbidden minors for non-separating toroidal graphs since they are all minors of the forbidden minors for toroidal graphs and we do not yet know the complete set of forbidden minors of toroidal graphs.

In Theorem 3, we also showed that there are maximal linklessly embeddable graphs with $3|V|-3$ edges. Now, a natural question that comes into mind is the following: Does every edge-maximal linklessly embeddable graph have at least $3 n-3$ edges? Negative answers to this question have recently been reported by $[1,11]$, using edge-maximal linklessly embeddable graphs with $\leqslant \frac{14}{5} n$ and $\leqslant \frac{25}{12} n$ edges respectively.

Theorem 3 also shows that there is a connection between non-separating planar graphs and linklessly embeddable graphs. It would be interesting to explore this connection further. In fact it was this connection that served as our first motivation for exploring the structure of non-separating planar graphs.

Another application of non-separating planar graphs is in decomposing planar graphs. In another paper, we use such a decomposition to prove a stronger version of the HananiTutte Theorem [7]. Finally, it would be interesting to see if there are other applications for non-separating planar graphs. 


\section{Acknowledgments}

The authors thank Peter Eades and David Wood for their helpful discussions and suggestions and the referee for a very careful reading of the paper and many helpful comments.

\section{References}

[1] M. Aires. On the number of edges in maximally linkless graphs. arXiv:1911.08552, 2019.

[2] D. Archdeacon. A Kuratowski theorem for the projective plane. Journal of Graph Theory, 5(3):243-246, 1981.

[3] D. Archdeacon, C. P. Bonnington, N. Dean, N. Hartsfield, and K. Scott. Obstruction sets for outer-cylindrical graphs. Journal of Graph Theory, 38(1):42-64, 2001.

[4] D. Archdeacon, N. Hartsfield, C. Little, and B. Mohar. Obstruction sets for outerprojective-planar graphs. Ars Combinatoria, 49:113-128, 1998.

[5] G. Chartrand and F. Harary. Planar permutation graphs. Annales de l'Institut Henri Poincaré. Probabilités et Statistiques, 3(4):433-438, 1967.

[6] J. H. Conway and C. McA. Gordon. Knots and links in spatial graphs. Journal of Graph Theory, 7(4):445-453, 1983.

[7] H. R. Dehkordi and G. Farr. A stronger version of the Hanani-Tutte Theorem. Submitted.

[8] C. Kuratowski. Sur le problème des courbes gauches en topologie. Fundamenta Mathematicae, 15(1):271-283, 1930.

[9] W. Mader. Homomorphiesätze für graphen. Mathematische Annalen, 178(2):154168, 1968.

[10] W. Myrvold and J. Woodcock. A large set of torus obstructions and how they were discovered. The Electronic Journal of Combinatorics, 25(1):\#P1.16, 2018.

[11] R. Naimi, A. Pavelescu, and E. Pavelescu. New bounds on maximal linkless graphs. arXiv:2007.10522, 2020.

[12] N. Robertson, P. Seymour, and R. Thomas. Sachs' linkless embedding conjecture. Journal of Combinatorial Theory, Series B, 64(2):185-227, 1995.

[13] N. Robertson and P. D. Seymour. Graph minors. XX. Wagner's conjecture. Journal of Combinatorial Theory, Series B, 92(2):325-357, 2004.

[14] N. Robertson, P. D. Seymour, and R. Thomas. A survey of linkless embeddings. In N. Robertson and P. D. Seymour, editors, Graph Structure Theory, pages 125-136. American Mathematical Society, 1991.

[15] H. Sachs. On a spatial analogue of Kuratowski's theorem on planar graphs - an open problem. In M. Borowiecki, J. W. Kennedy, and M. M. Sysło, editors, Graph Theory, pages 230-241. Springer, 1983.

[16] K. Wagner. Bemerkungen zum Vierfarbenproblem. Jahresbericht der Deutschen Mathematiker-Vereinigung, 46:26-32, 1936. 